FTUV-9830

IFIC-9830

hep-ph/9809376

\title{
Neutrino Conversions in Solar Random Magnetic Fields.
}

\author{
V.B Semikoz ${ }^{a, b}$, E. Torrente-Lujan ${ }^{b}$. \\ e-mail: torrente,semikoz@flamenco.ific.uv.es. \\ a The Institute of the Terrestrial Magnetism, the Ionosphere and Radio Wave \\ Propagation of the Russian Academy of Sciences \\ IZMIRAN, Troitsk, Moscow region, 142092, Russia \\ ${ }^{b}$ Instituto de Física Corpuscular - C.S.I.C., Departament de Física Teòrica \\ Universitat de València, 46100 Burjassot, València, Spain.
}

\begin{abstract}
We consider the effect of a random magnetic field in the convective zone of the Sun superimposed to a regular magnetic field on resonant neutrino spin-flavour oscillations. We argue for the existence of a field of strongly chaotic nature at the bottom of the convective zone. In contrast to previous attempts we employ a model motivated regular magnetic field profile: it is a static field, solution to the solar equilibrium hydromagnetic equations. These solutions has been known for a long time in the literature, we show for the first time that in addition they are twisting solutions. In this scenario electron antineutrinos are produced through cascades like $\nu_{e L} \rightarrow \nu_{\mu L} \rightarrow \tilde{\nu}_{e R}$, The detection of $\tilde{\nu}_{e R}$ at Earth would be a long-awaited signature of the Majorana nature of neutrino. The expected signals in the different experiments (SK,GALLEXSAGE,Homestake) are obtained as a function of the level of noise, regular magnetic field and neutrino mixing parameters. Previous results obtained for small mixing and ad-hoc regular magnetic profiles are reobtained. We confirm the strong suppression for large part of the parameter space of the $\tilde{\nu}_{e R}$-flux for high energy boron neutrinos in agreement with present data of the SK experiment.

We find that MSW regions $\left(\Delta m^{2} \approx 10^{-5} \mathrm{eV}^{2}\right.$, both small and large mixing solutions) are stable up to very large levels of noise $(\mathrm{P}=0.7-0.8)$ but they are acceptable from the point of view of antineutrino production only for moderate levels of noise $(P \approx 0.95)$.

For strong noise and reasonable regular magnetic field, any parameter region $\left(\Delta m^{2}, \sin ^{2} 2 \theta\right)$ is excluded. As a consequence, we are allowed to reverse the problem and to put limits on r.m.s field strength and transition magnetic moments by demanding a particle physics solution to the SNP under this scenario.
\end{abstract}

PACS codes: 13.10.+q; 13.15.-f; 13.40.Fn; 14.60.Gh; 96.60.Kx.

Key words: Neutrino, Magnetic moment, Magnetic fields, Reynolds number. 


\section{Introduction}

A neutrino transition magnetic moment can account, both for the observed deficiency of the solar neutrino flux and the time variations of the signal. The overall deficit is caused by the alteration or suppression of the neutrino energy spectrum. The time dependence may be caused by time variations of the magnetic field in the convective zone of the Sun. As it has been shown in [1], magnetic moments solutions are not ruled out by present experimental data; that is valid for both, absolute deficits and time variations of the observed solar neutrino flux.

In transverse magnetic fields, neutrinos with transition magnetic moments will experience spin and flavour rotation simultaneously (resonant spin-flavour precession, RSFP). The observation of electron antineutrinos from the Sun would lead to the conclusion that the neutrinos are Majorana particles. There are however stringent bounds on the presence of solar electron antineutrinos coming from the High energy Boron neutrinos ( $E_{\nu}>\approx 8.3 \mathrm{MeV}$ [2, 3]).

Magnetic fields measured on the surface of the Sun are weaker than within the interior of the convective zone. The mean field value over the solar disc is of the order of $1 \mathrm{G}$ and in the solar spots magnetic field strength reaches $1 \mathrm{KG}$. In magnetic hydrodynamics (MHD) one can explain such fields in a self-consistent way if these fields are generated by dynamo mechanism at the bottom of the convective zone. In this region the strength of small scale regular magnetic fields could reach a value of $100 \mathrm{KG}$. These fields propagate through the convective zone and photosphere decreasing in the strength value while increasing in the scale giving traces in from of loops in bipolar active regions (solar spots).

Large-scale toroidal magnetic field created by dynamo mechanism in convective zone has strength even less than small-scale r.m.s fields near the bottom of convective zone. This is the main reason why one should consider neutrino propagation in the random magnetic field of the Sun. The ratio of the r.m.s. random field and the regular (toroidal) field may be $\sim 20-50$, therefore the problem of RSFP neutrino propagation in noisy magnetic field seems to be important.

Estimations for the ratio of rms fields to regular field are necessarily very rough. In

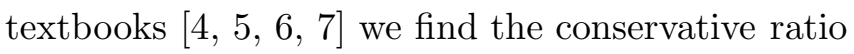

$$
\left\langle\tilde{B}^{2}\right\rangle / B_{0}^{2} \sim 1
$$

In more elaborate models the ratio of magnetic energy densities is given by the magnetic Reynolds number,

$$
\left\langle\tilde{B}^{2}\right\rangle / B_{0}^{2} \sim R_{m}^{\sigma}
$$

which may be much bigger than unity for plasma with large conductivity. Here $\sigma>0$ is a topology index [四, 步, 8, 9].

The effect of random magnetic fields in RSFP solutions to the SNP and antineutrino production has been explored previously for simplified models [10, 11]. In this work we will deal with the complete problem, we will present calculations of neutrino spin flavour conversions in presence of matter and magnetic field. The magnetic field will have two 
ingredients. The first ingredient will be a a theoretically motivated solar magnetic field profile. This magnetic field, which is one solution to the static magnetic hydrodynamic equations, is in addition a twisting field. The degree of twisting, the transversal profile and the ratio core field/ convection field are functionally related under this model. Of all possible solutions, in practical calculations we have considered only the solution with minimum twist which is at the same time the solution which implies a minimum value for the central field ( a factor 2 or less of the magnetic field at the convective zone). As a second ingredient, the effect of a layer of magnetic noise generated at the bottom of the convective zone has been included, we have justified that the level of noise in this region can be certainly very high.

Notice that an analogous problem but without the regular twist field motivated here while with use of a direct numerical method for dealing with the random magnetic fields in the solar convective zone has been recently considered ([12]). The results obtained in 12] are in general consistent with those obtained in the present work (see Conclusions below).

In Section (2) we present the effective Hamiltonian governing the evolution of the $4 \times 4$ neutrino wave function (two flavors times two helicities) in presence of a generic magnetic field. In Section (3.1) we give some physical arguments supporting the idea that r.m.s fields could be indeed important in the solar convective region. The analytical expressions for the hydrodynamic-inspired regular large-scale magnetic field appear in Section (3.2). There we show how different configurations of this field can be classified according to its intrinsic "twist" in the perpendicular plane to neutrino propagation. In Section (4) we give the master equation governing the time evolution of the averaged density matrix in presence of the random magnetic field. We show analytically how in the limit where chaotic field dominates, the $4 \times 4$ evolution equations decouples and the averaged transition probabilities follow a Markovian process. The results of the numerical integration of the exact averaged master equation are shown and discussed in Sections (5) and (6). From the calculated transition probabilities the expected total signals in each of the existing experiments (SK, SAGE-GALLEX,HOMESTAKE) are obtained and $\chi^{2}$ allowed parameter regions. In addition the expected electron antineutrino signal in SK is calculated and compared with existing bounds.

\section{The Master equation}

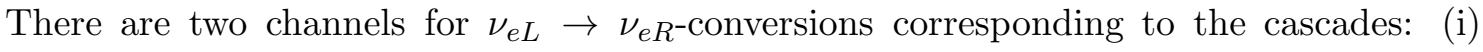
$\nu_{e L} \rightarrow \nu_{\mu L} \rightarrow \tilde{\nu}_{e R}$ or (ii) $\nu_{e L} \rightarrow \tilde{\nu}_{\mu R} \rightarrow \tilde{\nu}_{e R}$. The former case realizes if we assume a zero field in the radiative zone and in the core or if we exclude right-handed neutrino production there and the MSW-conversion $\left(\nu_{e L} \rightarrow \nu_{\mu L}\right)$ takes place before neutrino reaches convective zone. The latter case (ii) realizes when a strong magnetic field is present both in the radiative zone and in the solar core. In this case the RSFP takes place before the MSW-conversion since the $\nu_{e L}-\tilde{\nu}_{\mu R}$ energy splitting $V=G_{F} \sqrt{2}\left(N_{e}\left(r_{1}\right)-N_{n}\left(r_{1}\right)\right)$ is less than for the MSW one $\left(\nu_{e L} \rightarrow \nu_{\mu l}\right)$ at the same point $V=G_{F} \sqrt{2} N_{e}\left(r_{1}\right)$. This is true for 
the typical admixture of helium and heavy elements considered to be present in the inner layers of the Sun.

We consider conversions $\nu_{e L, R} \rightarrow \nu_{a L, R}, a=\mu$ or $\tau$, (for definiteness we will refer to $\mu$ for the rest of this work) for two neutrino flavors obeying the master evolution equation

$$
i \partial_{t}\left(\begin{array}{c}
\nu_{e L} \\
\bar{\nu}_{e R} \\
\nu_{\mu L} \\
\bar{\nu}_{\mu R}
\end{array}\right)=\left(\begin{array}{cccc}
V_{e}-c_{2} \delta & 0 & s_{2} \delta & \mu B_{\perp}^{+}(t) \\
0 & -V_{e}-c_{2} \delta & -\mu B_{\perp}^{-}(t) & s_{2} \delta \\
s_{2} \delta & -\mu B_{\perp}^{+}(t) & V_{\mu}+c_{2} \delta & 0 \\
\mu B_{\perp}^{-}(t) & s_{2} \delta & 0 & -V_{\mu}+c_{2} \delta
\end{array}\right)\left(\begin{array}{c}
\nu_{e L} \\
\bar{\nu}_{e R} \\
\nu_{\mu L} \\
\bar{\nu}_{\mu R}
\end{array}\right)
$$

where $c_{2}=\cos 2 \theta, s_{2}=\sin (2 \theta), \delta=\Delta m^{2} / 4 E$ are the neutrino mixing parameters; $\mu=\mu_{12}$ is the neutrino active-active transition magnetic moment;

$$
B_{\perp}^{ \pm}(t)=B_{0 \perp}^{ \pm}(t)+\tilde{B}_{\perp}^{ \pm}(t)
$$

is the magnetic field component which is perpendicular to the neutrino trajectory in the Sun;

$$
V_{e}(t)=G_{F} \sqrt{2}\left(\rho(t) / m_{p}\right)\left(Y_{e}-Y_{n} / 2\right)
$$

and

$$
V_{\mu}(t)=G_{F} \sqrt{2}\left(\rho(t) / m_{p}\right)\left(-Y_{n} / 2\right)
$$

are the neutrino vector potentials for $\nu_{e L}$ and $\nu_{\mu L}$ in the Sun given by the abundances of the electron $\left(Y_{e}=m_{p} N_{e}(t) / \rho(t)\right)$ and neutron $\left(Y_{n}=m_{p} N_{n}(t) / \rho(t)\right)$ components and by the SSM density profile 13

$$
\rho(t)=250 \mathrm{gcm}^{-3} \exp (-10.54 t) .
$$

The transverse magnetic field $B_{\perp}(t)$ appearing in Eq.(2) is given by the following expression where Cartesian and polar coordinates are written explicitly:

$$
B_{\perp}^{ \pm}(t) \equiv B_{x}(t) \pm i B_{y}(t) \equiv\left|B_{\perp}(t)\right| e^{ \pm i \Phi(t)}
$$

where

$$
\Phi(t)=\arctan B_{y} / B_{x} .
$$

The Cartesian form is useful in writing the averaged master equation. The polar form is the most convenient in separating out the twisting part.

The nature and magnitude for the regular $\left(B_{0 \perp}\right)$ and chaotic parts $\left(\tilde{B}_{\perp}\right)$ of the magnetic field will be the subject of the next section. The equation for the evolution of the average density matrix corresponding to the master equation (1) can be found using the formalism developed in [14] and will presented later in this this work.

The magnetic field strength enters the evolution Eq.(11) being multiplied by the neutrino transition moment $\mu$. The existing upper limits on the magnetic moment of the electron neutrino include the laboratory bound $\mu<3-4 \times 10^{-10} \mu_{B}$ from reactor experiments as well as stronger (one or two orders or magnitude) astrophysical and cosmological limits. In our calculations we will consider always the product $\mu B$. Expected values of $B \approx 1-100$ $\mathrm{kG}$ in the Sun convective zone and $\mu=10^{-11} \mu_{B}$ would give an expected range for the product $\mu B \approx 10^{-8}-10^{-6} \mu_{B} G \approx 5.610^{-17}-10^{-15} \mathrm{eV}$ or in the practical units which will be used throughout this work $\mu B \approx 0.1-10.0 \mu_{11} B_{4}$. 


\section{Solar magnetic fields}

\subsection{Random magnetic fields}

The r.m.s. random component $\sqrt{\left\langle\tilde{B}^{2}(t)\right\rangle}$ can be comparable with the regular one, $B_{0}(t)$, and maybe even much stronger than $B_{0}$, if a large magnetic Reynolds number $R_{m}$ leads to the effective dynamo enhancement of small-scale (random) magnetic fields.

Let us give simple estimates of the magnetic Reynolds number $R_{m}=l v / \nu_{m}$ in the convective zone for fully ionized hydrogen plasma $\left(T \gg I_{H} \sim 13.6 \mathrm{eV} \sim 10^{5} \mathrm{~K}\right)$. Here $l \sim 10^{8} \mathrm{~cm}$ is the size of eddy (of the order of magnitude of a granule size) with the turbulent velocity inside of it $v \sim v_{A} \sim 10^{5} \mathrm{~cm} / \mathrm{s}$ where $v_{A}=B_{0} / \sqrt{4 \pi \rho}$ is the Alfven velocity for MHD plasma, $B_{0}$ is a large-scale field in convective zone and $\rho$ is the matter density (in $\mathrm{g} / \mathrm{cm}^{3}$ ) in the SSM.

The magnetic diffusion coefficient $\nu_{m}=c^{2} / 4 \pi \sigma_{\text {cond }}(\sim$ magnetic viscosity) enters the diffusion term of the Faraday equation,

$$
\frac{\partial \vec{B}(t)}{\partial t}=\operatorname{rot}[\vec{v} \times \vec{B}(t)]+\nu_{m} \Delta \vec{B}
$$

Here $c$ is the light velocity; the conductivity of the hydrogen plasma $\sigma_{c o n d}=\omega_{p l}^{2} / 4 \pi \nu_{e p}$.

$$
\omega_{p l}=\sqrt{4 \pi e^{2} n_{e} / m_{e}}=5.65 \times 10^{4} \sqrt{n_{e}} s^{-1}
$$

is the plasma (Langmuir) frequency; $\nu_{e p}=50 n_{e} / T^{3 / 2} s^{-1}$ is the electron-proton collision frequency, the electron density $n_{e}\left(=n_{p}\right)\left(\mathrm{cm}^{-3}\right)$ and the temperature $T(\mathrm{~K})$.

Thus we find that the magnetic diffusion coefficient

$$
\nu_{m} \simeq 10^{13}(T / 1 K)^{-3 / 2} \mathrm{~cm}^{2} \mathrm{~s}^{-1}
$$

does not depend on the charge density $n_{e}$ and it is very small (the Reynolds number is big) for hot plasma $T \geq 10^{5} K \gg 1 K$. Actually, from the comparison of the first and second terms in the r.h.s. of the Faraday equation Eq. (6) we find that $v / l \gg \nu_{m} / l^{2}$, or $\nu_{m} \ll v l \sim 10^{13} \mathrm{~cm}^{2} s^{-1}$ since $T / 1 K \gg 1$. This means that the magnetic field in the Sun is mainly frozen-in. Neglecting the second term in Eq. (6) and using the Maxwell equation

$$
\operatorname{rot} \vec{E}=-c^{-1}(\partial \vec{B} / \partial t)
$$

we obtain the condition for frozen-in field: the Lorentz force vanishes,

$$
\sim(\vec{E}+[\vec{v} \times \vec{B}] / c) \approx 0
$$

but the current

$$
\vec{j}=\sigma_{\text {cond }}(\vec{E}+[\vec{v} \times \vec{B}] / c)
$$

remains finite if the conductivity is large, $\sigma_{\text {cond }} \rightarrow \infty$. 
The magnetic Reynolds number

$$
R_{m}=l v \omega_{p l}^{2} /\left(c^{2} \nu_{e p}\right) \simeq l v \times 10^{-13}(T / 1 K)^{3 / 2} \mathrm{~cm}^{2} s^{-1}
$$

is huge if we substitute the estimate $l v \sim 10^{13} \mathrm{~cm}^{2} s^{-1}$ given above. A large value for the Reynolds number is a necessary condition for an effective dynamo enhancement in the convective zone.

The estimation of the quantity $\eta$ for the solar convective zone (and other cosmic dynamos) is the matter of current scientific discussions. The most conservative estimate, simply based on equipartition, is $\eta=$ constant. According to direct observations of galactic magnetic field presumably driven by a dynamo, $\eta \approx 1.8$ [15]. A more developed theory of equipartition gives, say, $\eta \approx 4 \pi \ln R_{m}$ (see [16]). Notice that this estimate is considered now as very conservative. Basing on more detailed theories of MHD turbulence estimates like $b \sim \sqrt{R_{m}} B$ are discussed [8], [9].

The random magnetic field component in the Sun $(\langle\tilde{B}(t)\rangle=0)$ will be described in general by an arbitrary correlator

$$
\left\langle\tilde{B}(t) \tilde{B}\left(t^{\prime}\right)\right\rangle=\left\langle\tilde{B}^{2}\right\rangle f\left(t-t^{\prime}\right) .
$$

We will assume that the strength of the r.m.s. field squared $\left\langle\tilde{B}^{2}\right\rangle=\eta B_{0}^{2}$ is parametrized by the dimensionless parameter $\eta=R_{m}^{\sigma}>1$, which it can be, in general, much bigger than unity, $\eta \gg 1$.

The correlator function $f(t)$ is unknown a priori but it takes the particular $\delta$-correlator form $f(t)=L_{0} \delta(t)$ if the correlation length (for two neighboring magnetic field domains) is much less than the neutrino oscillation length, $L_{0} \ll l_{\text {osc }}$. $L_{0}$ can be considered a free parameter ranging in the interval $1-10^{4} \mathrm{~km}$. In the averaged evolution equations it appears only the product $\eta L_{0}$. Thus in what follows we will present our results as a function of the quantity $\mathrm{P}$ which is a simple function of such product:

$$
\begin{aligned}
P & =\frac{1}{2}(1+\exp (-\gamma)) \\
\gamma & \equiv \frac{4}{3} \Omega^{2} \Delta t \equiv \frac{4}{3} \eta L_{0}\left(\mu B_{0}\right)^{2} \Delta t
\end{aligned}
$$

The reason for using $P$ is that it is a good approximation for the depolarization that the presence of noise induces in the averaged neutrino density matrix. $\Delta t$ is the distance over which the noise is acting. We have supposed in our computations that the noise is effective only in a thin layer with thickness $\Delta t=0.1 R_{\odot}$ starting at $r=0.7 R_{\odot}$, the bottom of the convective zone. This is represented together with the regular transverse profile in Fig.(11). In Table (2) the quantities $\sqrt{\left\langle B_{0}^{2}\right\rangle}$ and $\eta$ are computed for a given $P$ supposing the reasonable value $L_{0}=1000 \mathrm{Km}$.

\subsection{Regular large-scale magnetic field in the Sun. Twist field.}

Many phenomenological formulas for $B_{0 \perp}$ in the convective zone and in the central region of the Sun [17], with or without twist, has been employed in the literature. In particular, 
twist was applied for effective enhancement of the process $\nu_{e L} \rightarrow \tilde{\nu}_{e R}$ in the Sun (i.e. in Ref.[18]). Without twist the coherent sum of two amplitudes for the process $\nu_{e L} \rightarrow \tilde{\nu}_{e R}$,

$$
M_{1}\left(\nu_{e L} \rightarrow \nu_{\mu L} \rightarrow \tilde{\nu}_{e R}\right)+M_{2}\left(\nu_{e L} \rightarrow \tilde{\nu}_{\mu R} \rightarrow \tilde{\nu}_{e R}\right),
$$

occurs proportional to the small abundance of neutrons and production of electron antineutrino is negligible [1].

In the presence of the twist, $\Phi(t) \neq 0$, this sum becomes proportional to the angular velocity

$$
\dot{\Phi}=\kappa R_{\odot}^{-1}=\kappa \times 0.3 \times 10^{-15} \mathrm{eV}
$$

where $[\kappa]=n=1,2, \ldots$ is the effective number of revolutions of the field $\vec{B}_{\perp}$ in the plane which is perpendicular to the neutrino trajectory. The more rounds $(n \gg 1)$ take place before the resonant RSFP position, given by the condition

$$
G_{F} \sqrt{2}\left(N_{e}-N_{n}\right)-2 \delta \cos 2 \theta-\dot{\Phi}=0,
$$

the deeper the resonant point happens (for successive (positive) sign of $\dot{\Phi}$ ). It is possible then the merging of $\operatorname{RSFP}\left(\nu_{\mu L} \rightarrow \tilde{\nu}_{e R}\right)$ and MSW $\left(\nu_{e L} \rightarrow \nu_{\mu L}\right)$ resonances since the MSW resonance is unchanged, $\left(G_{F} \sqrt{2} N_{e}=2 \delta \cos 2 \theta\right)$. For the case of merging of resonances the of $\nu_{e L} \rightarrow \tilde{\nu}_{e R^{-c o n v e r s i o n s ~}}$ tend to be more adiabatical and complete than for separated resonances [19].

Unfortunately, in the known MHD plasma solutions for the toroidal field evolving in convective zone due to dynamo mechanism (Yoishimura model, 20]) the predicted number of revolutions for twist (around toroids in northern and southern hemispheres of the Sun) is very small: $n \gtrsim 1$. For this small twist rate $\dot{\Phi} \sim n$ the resonances above are fulfilled for a small $\Delta m^{2} \sim 10^{-7}-10^{-8} e V^{2}$ and happen not too deep in solar interior to have important consequences.

Below we apply for the neutrino conversions described by our master equation Eq. (1) the self-consistent model of large-scale regular field given in [21]. The global solar magnetic field is the axisymmetric equilibrium solution of the MHD static equations (quiet Sun) in the spherically symmetric gravitational field of the Sun. The reasonable boundary condition $B_{0}=0$ on the photosphere $\left(r=R_{\odot}\right)$ is imposed in addition. Any field solution to these equations and boundary conditions is a twisting field with an arbitrary small or large number of revolutions along radius $(k=1, \ldots, \infty$, the twist rate can be taken as label for distinguish particular solution within the family).

The spherical components of the magnetic field for the fundamental radial mode $n=1$ do not depend on azimuthal angle $\phi$. In the whole region $0 \leq r \leq R_{\odot}$ they are of the form (the different solutions are labeled by $k$ ):

$$
\begin{aligned}
B_{0 r}\left(r, \theta_{s}\right) & =2 K \cos \theta_{s}\left[1-\frac{3 R_{\odot}^{2}}{r^{2} z_{2 k} \sin 2 z_{2 k}}\left(\frac{\sin \alpha r}{\alpha r}-\cos \alpha r\right)\right], \\
B_{0 \theta_{s}}\left(r, \theta_{s}\right) & =-K \sin \theta_{s}\left[2+\frac{3 R_{\odot}^{2}}{r^{2} z_{2 k} \sin 2 z_{2 k}}\left(\frac{\sin \alpha r}{\alpha r}-\cos \alpha r-\alpha r \sin \alpha r\right)\right], \\
B_{0 \phi}\left(r, \theta_{s}\right) & =K z_{2 k} \sin \theta_{s}\left[\frac{r}{R_{\odot}}-\frac{3 R_{\odot}^{2}}{r z_{2 k} \sin 2 z_{2 k}}\left(\frac{\sin \alpha r}{\alpha r}-\cos \alpha r\right)\right] .
\end{aligned}
$$


Here $\theta_{s}$ is the polar angle. $z_{2 k}$ is any root of the spherical Bessel function

$$
f_{n}(z)=\sqrt{z} J_{n+1 / 2}(z)
$$

of the first order $(n=1)$. This equation follows from the boundary condition. For the first three roots:

$$
z_{2 k} \alpha R_{\odot}=5.7635,9.0950,12.3224, \ldots(k=1,2,3, \ldots) .
$$

The constant $K$ is related with central field $B_{\text {core }}$, the only free parameter in this model:

$$
K=\frac{B_{\text {core }}}{2\left(1-\alpha R_{\odot} / \sin \alpha R_{\odot}\right)} .
$$

The modulus of the perpendicular component is of the form:

$$
B_{0 \perp}=\sqrt{B_{0 \phi}^{2}+B_{0 \theta_{s}}^{2}}=B_{\text {core }} \frac{\sin \theta}{r} f(r)
$$

where $f(r)$ is some known function of gentle behavior. Obviously, on the solar equator $\left(\theta_{s}=0\right)$ the perpendicular component vanishes. This is the same behavior as it is shown by the toroidal field in the Yoishimura model [20] $\mathrm{I}$.

Notice the regular behavior of $\vec{B}_{0}$ at the Sun center $(r=0)$ :

$$
\begin{aligned}
B_{r}(0) & =B_{\text {core }} \cos \theta_{s} \\
B_{\theta_{s}}(0) & =-B_{\text {core }} \sin \theta_{s} \\
B_{\phi}(0) & =B_{\text {core }} \sin \theta_{s} r / R_{\odot} \rightarrow 0 .
\end{aligned}
$$

The twist for the perpendicular component $B_{0 \perp}(r)$ is defined by the angle:

$$
\begin{aligned}
& \Phi(r)_{z 2 k}=\arctan B_{\phi}(r) / B_{\theta}(r) \sim z_{2 k} \\
& \dot{\Phi}(r)_{z_{2 k}} \approx k R_{\odot}^{-1} .
\end{aligned}
$$

For $k=1$ for example, $\Phi$ changes between 0 and $\pi$ along the neutrino path from the core of the Sun through the surface at an uniform rate. For higher roots we observe that effectively $\dot{\Phi} \approx k \pi$ uniformly.

According to this model, the expected magnetic field at the core is typically only 23 times (or less) the magnetic field at the convective zone. For the values that we will consider later, $B_{0.7} \approx<100-200 \mathrm{kG}$, the values corresponding at the core are well below astrophysical bounds derived from traces of these fields at solar surface.

\footnotetext{
${ }^{1}$ This property is general and could lead to semiannual variations of the neutrino flux if one takes into account inclination $\left(7^{\circ}\right)$ of the solar axis (perpendicular to solar equator) to the ecliptic with the Earth orbit.
} 


\section{The averaged master equation.}

The master Equation (1) can be written in terms of the density matrix $\rho(t)$ as:

$$
i \partial_{t} \rho=\left[H_{r e g}, \rho\right]+\mu \tilde{B}_{x}(t)\left[V_{x}, \rho\right]+\mu \tilde{B}_{y}(t)\left[V_{y}, \rho\right] .
$$

The elements of the matrices $H_{0}, V_{x}, V_{y}$ can be read off the Eq. (11). The $\tilde{B}_{x}, \tilde{B}_{y}$ are the Cartesian transversal components of the chaotic magnetic field. Vacuum mixing terms and matter terms corresponding to the SSM density profile given before and the regular magnetic part Hamiltonian, with the profile determined by the Eqs.(3), are all included in $H_{\text {reg }}$. In particular, the matrices $V_{x, y}$ are given in terms of the Pauli matrices $\sigma_{1,2}$ by:

$$
V_{x}=\left(\begin{array}{cc}
0 & i \sigma_{2} \\
-i \sigma_{2} & 0
\end{array}\right), \quad V_{y}=\left(\begin{array}{cc}
0 & -i \sigma_{1} \\
i \sigma_{1} & 0
\end{array}\right)
$$

It is our objective in this section to write the differential evolution equation for the average density matrix $\langle\rho\rangle$.

We assume that the components $\tilde{B}_{x}, \tilde{B}_{y}$ are statically independent, each of them characterized by a delta-correlation function:

$$
\begin{aligned}
\left\langle\tilde{B}_{x, y}(t) \tilde{B}_{x, y}\left(t^{\prime}\right)\right\rangle & =\left\langle\tilde{B}_{x, y}^{2}\right\rangle L_{0} \delta\left(t-t^{\prime}\right) \\
\left\langle\tilde{B}_{x}(t) \tilde{B}_{y}\left(t^{\prime}\right)\right\rangle & =0
\end{aligned}
$$

From now on we will make the following assumption, which is reasonable from equipartition arguments,

$$
\left\langle\tilde{B}_{x}^{2}\right\rangle=\left\langle\tilde{B}_{y}^{2}\right\rangle=\left\langle\tilde{B}_{0 \perp}^{2}\right\rangle / 2=\left\langle\tilde{B}_{0}^{2}\right\rangle / 3
$$

The averaged evolution equation is a simple generalization (see Ref. [14] for a complete derivation) of the well known Redfield equation [22, 23] for two independent sources of noise and reads $\left(\Omega^{2} \equiv L_{0} \mu^{2}\left\langle\tilde{B}^{2}\right\rangle / 2 \equiv \eta L_{0}\left(\mu B_{0}\right)^{2} / 3\right)$ :

$$
i \partial_{t}\langle\rho\rangle=\left[H_{r e g},\langle\rho\rangle\right]-i \Omega^{2}\left[V_{x},\left[V_{x},\langle\rho\rangle\right]\right]-i \Omega^{2}\left[V_{y},\left[V_{y},\langle\rho\rangle\right]\right] .
$$

It is possible to write the Eq.(21) in a more evolved form. Taking into account the particular form of the matrices $V_{x, y}$ we can simplify considerably the double commutators:

$$
i \partial_{t}\langle\rho\rangle=\left[H_{r e g},\langle\rho\rangle\right]-2 i \Omega^{2}\left(2\langle\rho\rangle-V_{x}\langle\rho\rangle V_{x}-V_{y}\langle\rho\rangle V_{y}\right) .
$$

The second term in Eq.(22), which gives the leading noise behavior, can be eliminated by a rescaling of the density matrix:

$$
\langle\rho(t)\rangle=\exp \left(-4 \Omega^{2} t\right)\left\langle\rho^{\prime}(t)\right\rangle .
$$

With this redefinition the evolution equation reads:

$$
i \partial_{t}\left\langle\rho^{\prime}\right\rangle=\left[H_{\text {reg }},\left\langle\rho^{\prime}\right\rangle\right]+i 2 \Omega^{2}\left(V_{x}\left\langle\rho^{\prime}\right\rangle V_{x}+V_{y}\left\langle\rho^{\prime}\right\rangle V_{y}\right)
$$


The expression in Eq.(23) is the one which has been used in the numerical calculations to be presented below. It is useful however to consider the solution to Eq. (23) when $H_{r e g} \equiv 0$. This is the appropriate limit when dealing with extremely low $\Delta m^{2}$ or very large energies, for an extreme level of noise or when the distance over which the noise is acting is small enough to consider the evolution given by $H_{r e g}$ negligible. In any other scenario it can give at least an idea of the general behavior of the solutions to the full Eq.(23). When $H_{0}=0$ only the two last terms in the equation remain and an exact simple expression is obtainable by ordinary algebraic methods. The full $4 \times 4$ Hamiltonian decouples in $2 \times 2$ blocks. The quantities of interest, the averaged transition probabilities, are given by the diagonal elements of $\langle\rho\rangle$. If $P_{f, i}$ are the final and initial probabilities ( at the exit and at the entrance of the noise region) their averaged counterparts fulfill linear relations among them, schematically:

$$
Q_{f}^{A, B}=M Q_{i}^{A, B}
$$

with $Q^{A, B}$ any of the two dimensional vectors

$$
Q^{A}=\left(\begin{array}{c}
\left\langle P\left(\nu_{e L} \rightarrow \nu_{e L}\right)\right\rangle \\
\left\langle P\left(\nu_{e L} \rightarrow \tilde{\nu}_{\mu R}\right)\right\rangle
\end{array}\right) \quad Q^{B}=\left(\begin{array}{c}
\left\langle P\left(\nu_{e L} \rightarrow \tilde{\nu}_{e R}\right)\right\rangle \\
\left\langle P\left(\nu_{e L} \rightarrow \nu_{\mu L}\right)\right\rangle
\end{array}\right)
$$

and the Markovian matrix $M$ :

$$
M=\left(\begin{array}{cc}
P & 1-P \\
1-P & P
\end{array}\right)
$$

with $P$ defined in Eq.(8). It can be shown that in this simple case $P$ is exactly the final polarization of the density matrix. In the general case with a finite $H_{\text {reg }}$ it can be shown numerically that the quantity $P$ still gives a reasonable approximation $(<10 \%)$ to the real polarization, at least for the cases of interest in this work.

\section{$5 \quad$ Results and Discussion.}

The present status of the Solar neutrino problem represented by the ratios between the solar neutrino observations and the solar model predictions is summarized in Table (1) of Ref.([24]) which for the sake of completeness we reproduce in (11). It is claimed in addition by a variety of analysis (check Ref. 225] for an useful review) that Homestake, the chlorine radiochemical experiment, presents an anticorrelation with the solar activity. Signal time variations are not observed by the Kamiokande experiment. The allowed time variation is restricted by to be $\approx<30 \%$ at $90 \%$ C.L. [26, 24].

We have calculated the expected neutrino signals in the Homestake, Ga-Ge and (Super)Kamiokande experiments. For this objective, the time averaged survival and transition probabilities have been obtained by numerical integration of the ensemble averaged master equation (17) for a regular magnetic profile $B_{0 \perp}$ as given in Eqs.(9),11) for $k=1$ and the matter density given by Eq.(阴). 
The free parameters of our model are four: the squared mass differences $\left(\delta=\Delta m^{2} / 2 E\right)$, the flavour mixing angle $\left(s_{2}^{2} \equiv \sin ^{2} 2 \theta\right)$, a noise strength parameter $(P)$ and the product of magnetic field and moment $\left(\mu B_{0 \perp}\right.$ at a given radius and latitude). We have found convenient to use the magnitude of the transverse magnetic field at the bottom of the convective zone $\left(r=0.7 R_{\odot}\right)$ for a solar latitude $\theta_{s}=7^{\circ}$; for other latitudes they have to be rescaled accordingly.

In Figs.(2) some illustrative cases. In Fig.(2) the dependence of the probabilities with $B_{0 \perp}$ for a fixed small mixing angle $\left(s_{2}^{2}=0.01\right)$ and in absence of noisy magnetic field is shown. In general spin-flavour precession is suppressed by large mass differences, the non-suppression condition is $\mu B>\approx \delta$. One can observe the effect of this suppression around $\delta \approx 10^{-7}-$ $10^{-8} \mathrm{eV}^{2} / \mathrm{MeV}$. Magnetic precession and MSW transitions respectively predominate below and above this value. For the range of values considered in this case we observe a very modest production of $\bar{\nu}_{e}$ 's (always smaller $<\approx 0.5 \%$ ). The production of $\bar{\nu}_{\mu}$ 's can be however very important. In Fig.(3) we show the dependence with $s_{2}^{2}$ for a fixed magnetic field of very low magnitude $\left(\mu B=1.0 \mu_{11} B_{4} \equiv 10^{-11} \mu_{B} \times 10 \mathrm{kG}\right)$. We observe again the same transition region around $\delta \approx 10^{-7}-10^{-8} \mathrm{eV}^{2} / \mathrm{MeV}$, below this range the magnetic precession dominates but for this low value of the magnetic field the conversion probability is anyway very small. No significant quantity of either electron or muon antineutrinos is produced irrespective of the mixing angle. For stronger magnetic fields (of the order $\left.\mu B \approx 15 \mu_{11} B_{4}\right)$ the results are similar, the triangular regions, distinctive structure of the MSW effect, appear strongly distorted, the production of muon antineutrinos is notable for $\delta \approx 10^{-6}-10^{-9} \mathrm{eV}^{2} / \mathrm{MeV}$ with mixing angles $s_{2}^{2}<0.6-0.7$. The production of electron antineutrinos is not significant even at these high magnetic fields.

In the next two figures we show the expected signals in the Chlorine, Gallium and Kamiokande experiments for a variety of parameter combinations and fixing the transverse magnetic field to a relative high value: $\mu B=15 \mu_{11} B_{4}$. Figs.(4, (4) correspond respectively to the cases $\mathrm{P}=1.00$ (absence of magnetic noise), $\mathrm{P}=0.70$ (strong noise). The signals in each of the experiments have been normalized to the value in absence of neutrino oscillations. Comparing both figures we can observe in the noisy case how the values for the signal approach $1 / 2$. This will be important later when we compute the parameter areas consistent with observed total rates, this smoothening, particularly in the Ga-Ge experiment will be the responsible for the appearance of allowed regions at high magnetic fields.

For the Kamiokande experiment we have taken into account the four elastic scattering processes $\nu_{x} e \rightarrow \nu_{x} e$ with $\nu_{x}=\nu_{e}, \nu_{\mu}, \bar{\nu}_{e}, \bar{\nu}_{\mu}$. Using the expressions for the elastic cross sections appearing in [27] we have defined an "effective" conversion probability:

$$
\begin{aligned}
P_{e f f, e} & =\sum_{x} c_{x} P_{\nu_{e} \nu_{x}} \\
c_{x} & =\sigma_{x}\left(E_{\nu}, T_{e, \text { min }}\right) / \sigma_{\nu_{e}}\left(E_{\nu}, T_{e, \text { min }}\right)
\end{aligned}
$$

The coefficients $c_{x}$ depend on $T_{e, \text { min }}$, the kinetic energy threshold for the observation of the scattered electron. For $T_{e, \text { min }} \approx 0 \mathrm{MeV}$ the coefficients $c_{x}$ are very accurately 
independent of the neutrino energy $E_{\nu}$ for values above $\approx 1 \mathrm{MeV}$. For $T_{e, \min } \approx 7 \mathrm{MeV}$ the coefficients $c_{x}$ associated with the muon neutrino and antineutrino are still practically energy independent while $c_{\bar{\nu}_{e}}$ is only slightly energy dependent.

The effective conversion probability is defined in such a way that the signal expected at the Super-Kamiokande experiment is:

$$
S_{S K}=N \int d E \epsilon(E) \sigma(E)_{\nu_{e}} \Phi(E) P_{e f f, e}(E)
$$

where $\epsilon(=1$. for us $)$ is the experimental detection efficiency, $\sigma_{\nu_{e}}$ the electron neutrinoneutrino elastic cross section and $\Phi$ the total incoming electron neutrino flux predicted by SSM. $\mathrm{N}$ is a normalization constant.

The weighted electron antineutrino appearance probability is defined as:

$$
\left\langle P_{\nu_{e} \bar{\nu}_{e}}\right\rangle=\frac{\int_{E>E t h} d E \sigma(E) \Phi(E) P_{\nu_{e}, \bar{\nu}_{e}}(E)}{\int_{E>E t h} d E \sigma(E) \Phi(E)}
$$

where $\sigma(E), \Phi(E)$ are respectively the differential cross section for the process isotropic background $\bar{\nu}_{e}+p \rightarrow e^{+} n$ and the differential total neutrino flux coming from the Sun according to the SSM [13]. The threshold energy has been chosen as $E_{t h}=9 \mathrm{MeV}$. From Kamiokande data we have the bound $\left\langle P_{\nu_{e} \bar{\nu}_{e}}\right\rangle=\approx 0.05$ [2, 3, 17].

In Figs.(6-9) we present the $\left(\Delta m^{2}, \sin ^{2} 2 \theta\right)$ exclusion plots from a combined $\chi^{2}$ analysis of the three experiments corresponding to the expected signals showed previously.

First we comment the results in complete absence of noise $(\mathrm{P}=1)$ which are represented in Fig.(6). For negligible or low regular magnetic field we observe the high squared mass difference solutions proportioned by the matter MSW effect. As the magnitude of the magnetic field increases new solutions appear and disappear in a complicated manner. The high angle MSW solution rests practically unmodified for all the range considered. The low angle solution however disappears at high magnetic fields, after experiencing some distortion coming from its merging with newborn magnetic solutions (compare low angle allowed regions in Plots (B) and (C)). The antineutrino production (dashed lines) is in general low and Kamiokande bounds are not specially restrictive except at very high magnetic fields. Note in Plot (D) the very different behavior of the two existing allowed regions: while the in MSW region the antineutrino production is in the $0.1-1 \%$, compatible comfortably with Kamiokande bounds, the RSFP solution reach a value well above $10 \%$ and is excluded by them. It seems apparent that there are acceptable particle solutions to the SNP even for very large regular magnetic fields.

The pattern of the electron antineutrino probability is very different when a small level of noise $(\mathrm{P}=0.95$, Fig.(7) $)$ is switched on. For $\Delta m^{2}>10^{-6} \mathrm{eV}^{2}$ : the antineutrino iso-probability lines follow the characteristic MSW triangular patterns in this region. The structure of the allowed regions remain unmodified. The electron antineutrino yield in these regions is below the $5 \%$ level for all cases except in the same region at high mixing angle and low squared mass difference as before. Note that only low mixing angle solutions with moderate regular magnetic fields would be acceptable if future data situate the antineutrino bound at the $1-3 \%$ level. 
For stronger levels of noise $(\mathrm{P}=0.8$, Figs. (8) $)$ the same comments can be said. The structure and position of the allowed regions from combined total rates are practically unmodified but the antineutrino yield impose strong restrictions. For an antineutrino probability smaller than $3 \%$ only some residual, $90 \%$ C.L., allowed regions exist at very small mixing angle, $\Delta m^{2} \approx 10^{-6}-10^{-7} \mathrm{eV}^{2}$ and moderately high regular magnetic field (Plot (C)). The same regions are still acceptable for $\mathrm{P}=0.7$, Figs.(9)). For this level of noise something unexpected happens at extremely high regular magnetic field (Plot (D)) (probably too high to be acceptable on astrophysical grounds): a new, large, acceptable region appear for $\Delta m^{2} \approx 10^{-5}$. This region disappears again for extremely high chaotic fields $(\mathrm{P}=0.55$, Figs. (10) $)$. This apparently erratic behavior could be worthy of a more detailed further study. Note that even for this value of $\mathrm{P}$ some residual regions with a $90 \%$ C.L. are marginally acceptable from reconciliation of all experiment total rates and antineutrino bounds [2] if the regular magnetic field is $\approx 200 \mathrm{kG}$ ( for $\left.\mu=10^{-11} \mu_{B}\right)$.

\section{Conclusions.}

We have presented calculations of neutrino spin flavour precession in presence of matter and magnetic field for a theoretically motivated solar magnetic field profile. This magnetic field, which is the solution to static magnetic hydrodynamic equations, is a twisting field. The degree of twisting and the transversal profile are functionally related under this model. In our calculations we have considered only the solution with minimum twisting.

Additionally the effect of a layer of magnetic noise at the bottom of the convective zone has been considered, we have justified that the level of noise in this region can be certainly very high.

We have presented expected signals and expected production of antineutrinos with a without presence of noise. We confirm previous results [17, 25] for small mixing and ad-hoc regular magnetic field profiles.

We find that MSW regions $\left(\Delta m^{2} \approx 10^{-5} \mathrm{eV}^{2}\right.$, both small and large mixing solutions) are stable up to very large levels of noise $(\mathrm{P}=0.7-0.8)$ but they are acceptable from the point of view of antineutrino production only for moderate levels of noise $(P \approx 0.95)$.

This is in agreement with recent results obtained through a direct ensemble averaging of the solution to Eq.(11) [12]. The stronger r.m.s field occurs at the convective zone, the wider $\left(\delta m^{2}, \sin ^{2} 2 \theta\right)$ region should be excluded when considering the constrain imposed by existing antineutrino bounds.

For strong noise, $P=0.7$ or bigger and reasonable regular magnetic field, any parameter region $\left(\Delta m^{2}, \sin ^{2} 2 \theta\right)$ is excluded. This model of noisy magnetic field is not compatible with particle physics solutions to the SNP. One is allowed then to reverse the problem and to put limits on r.m.s field strength, correlation length and transition magnetic moments by demanding a solution to the SNP under this scenario.

\section{Acknowledgments}


The authors thank Sergio Pastor and Jose Valle for fruitful discussions. V.B. Semikoz has been supported by RFFR grant 97-02-16501 and by INTAS grant 96-0659 of the European Union. E. Torrente-Lujan has been supported by DGICYT under Grant PB951077 and by a DGICYT-MEC contract at Univ. de Valencia. 


\section{References}

[1] E. Kh. Akhmedov, Phys. Lett. B255 (1991) 84.

[2] G. Fiorentini, M. Moretti, F.L. Villante. hep-ph/9707097 .

[3] R. Barbieri, G. Fiorentini, G. Mezzorani and M. Moretti. Phys. Lett. B259 (1991) 119 .

[4] S.I. Vainstein, A.M. Bykov, I.M. Toptygin. Turbulence, Current Sheets and Schocks in Cosmic Plasma. Gordon and Breach, 1993.

[5] S.I. Vainshtein, Y.B. Zeldovich and A.A. Ruzmakin, Turbulent dynamo in Astrophysics. Nauka, Moscow, 1980.

[6] E. N. Parker. Cosmical Magnetic Fields. Clarendon Press, Oxford, 1979.

[7] E. N. Parker. Astrophys. J., 408 (1993) 707.

[8] S.I. Vainstein, F. Cattaneo, 1992, Astrophysical Journal, 393 (1992) 165.

[9] A.V. Gruzinov, P.H. Diamond, 1994, Phys. Rev. Lett., 72 (1994) 1651.

[10] E. Torrente-Lujan. hep-ph/9807371.

[11] E. Torrente-Lujan. hep-ph/9807426.

[12] A.A. Bykov, V.Y. Popov, A.I. Rez, V.B. Semikoz, D.D. Sokoloff. hep-ph/9808342.

[13] J.N. Bahcall and M.H. Pinsonneault, Rev. Mod. Phys. 67 (1995) 781.

[14] E. Torrente-Lujan. hep-ph/9807361.

[15] A.A. Ruzmaikin, A.M. Shukurov, D.D. Sokoloff, Magnetic fields of Galaxies, Kluwer, Dordrecht, 1988.

[16] Ya.A. Zeldovich, A.A. Ruzmaikin, D.D. Sokoloff, Magnetic fields in astrophysics, Gordon and Breach, N.Y., 1983

[17] E.K. Akhmedov, A. Lanza, S.T. Petcov. Phys. Lett., B 303 (1993) 85-94.

[18] A.B. Balantekin and F. Loreti, Phys. Rev. D48 (1993) 5496.

[19] E.K. Akhmedov, S.T. Petcov, A.Y. Smirnov. Phys. Rev. D48 (1993) 2167.

[20] H. Yoishimura, Astrophys. J., 178 (1972) 863; Astrophys. J. Suppl. Ser. 52 (1983) 363.

[21] V.A. Kutvitskii and L.S. Solov'ev, Sov. Phys. JETP. 78 (1994) 456. 
[22] F.N. Loreti and A.B. Balantekin, Phys. Rev. D50 (1994) 4762-4770.

[23] E. Torrente-Lujan. hep-ph/9602398.

[24] J.N. Bahcall, P.I. Krastev and A.Y. Smirnov, hep-ph/9807216.

[25] E.Kh. Akhmedov. hep-ph/9705451.

[26] Y. Suzuki (Kamiokande Collaboration), Talk given at the 6th International Workshop on Neutrino Telescopes, Venice, February 22-24,1994.

[27] S. Pastor, V.B. Semikoz, J.W.F. Valle. Phys. Lett. B369 (1996) 301-307. 


\begin{tabular}{|c|c|c|c|}
\hline Experiment & Data \pm (stat.) \pm (syst.) & Theory & $S_{\text {Data }} / S_{S S M}$ \\
\hline Homestake & $2.56 \pm 0.16 \pm 0.14$ & $7.7_{-1.0}^{+1.2} \mathrm{SNU}$ & $0.33 \pm 0.029$ \\
Kamiokande & $2.80 \pm 0.19 \pm 0.33$ & $5.15_{-0.7}^{+1.0} 10^{6} \mathrm{~cm}^{-2} \mathrm{~s}^{-1}$ & $0.54 \pm 0.07$ \\
SAGE & $66.6_{-8.1}^{+7.8}$ & $129_{-6}^{+8} \mathrm{SNU}$ & $0.52 \pm 0.06$ \\
GALLEX & $77.5 \pm 6.2_{-4.7}^{+4.3}$ & $129_{-6}^{+8} \mathrm{SNU}$ & $0.60 \pm 0.06$ \\
SK (504 days) & $2.44_{-0.09-0.06}^{+0.05+0.07}$ & $5.15_{-1.12}^{+0.93} 10^{6} \mathrm{~cm}^{-2} \mathrm{~s}^{-1}$ & $0.474 \pm 0.020$ \\
\hline
\end{tabular}

Table 1: Neutrino event rates measured by solar neutrino experiments, and corresponding predictions from the SSM (see Ref. [24] and references therein, we take the INT normalization for the SSM data). The quoted errors are at $1 \sigma$.

\begin{tabular}{|c|c|c|c|c|}
\hline $\mathrm{P}$ & $\sqrt{\left\langle B_{0}^{2}\right\rangle}$ & $\eta,(\mu B=1)$ & $\eta,(\mu B=5)$ & $\eta,(\mu B=20)$ \\
\hline 1.00 & 0.0 & 0.0 & 0.00 & 0.00 \\
0.999 & 2.2 & 5.1 & 0.2 & 0.01 \\
0.95 & 10 & 100 & 4 & 0.2 \\
0.80 & 23 & 540 & 22 & 1.3 \\
0.70 & 34 & 1100 & 46 & 3.0 \\
0.55 & 51 & 2600 & 100 & 6.6 \\
0.51 & 68 & 4600 & 180 & 11 \\
\hline
\end{tabular}

Table 2: Values for the noise parameters $\sqrt{\left\langle B_{0}^{2}\right\rangle}$ and $\eta$ (Eq.(8)) assuming $L_{0}=1000 \mathrm{~km}$. All $\mu B$ are given in $\mu_{11} B_{4}$ units. The quantities $P, \eta$ are dimensionless. 


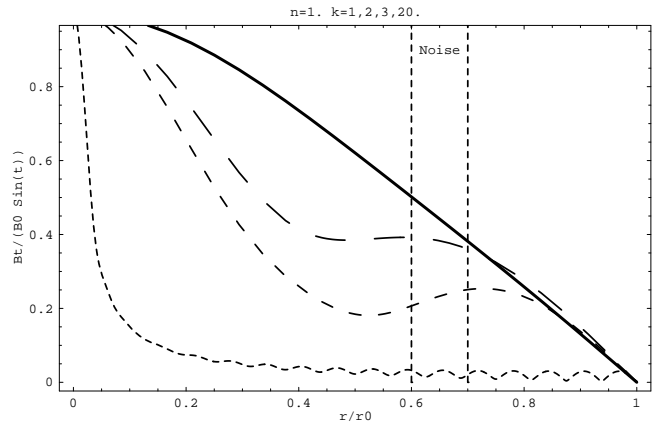

Figure 1: Transversal magnetic field profile. Solutions $B_{0 \perp}=B_{0} \sin \theta f(r) / r$ (see Eq.(11)) with $k=1$ (Continuos line) 2,3,30 respectively. 

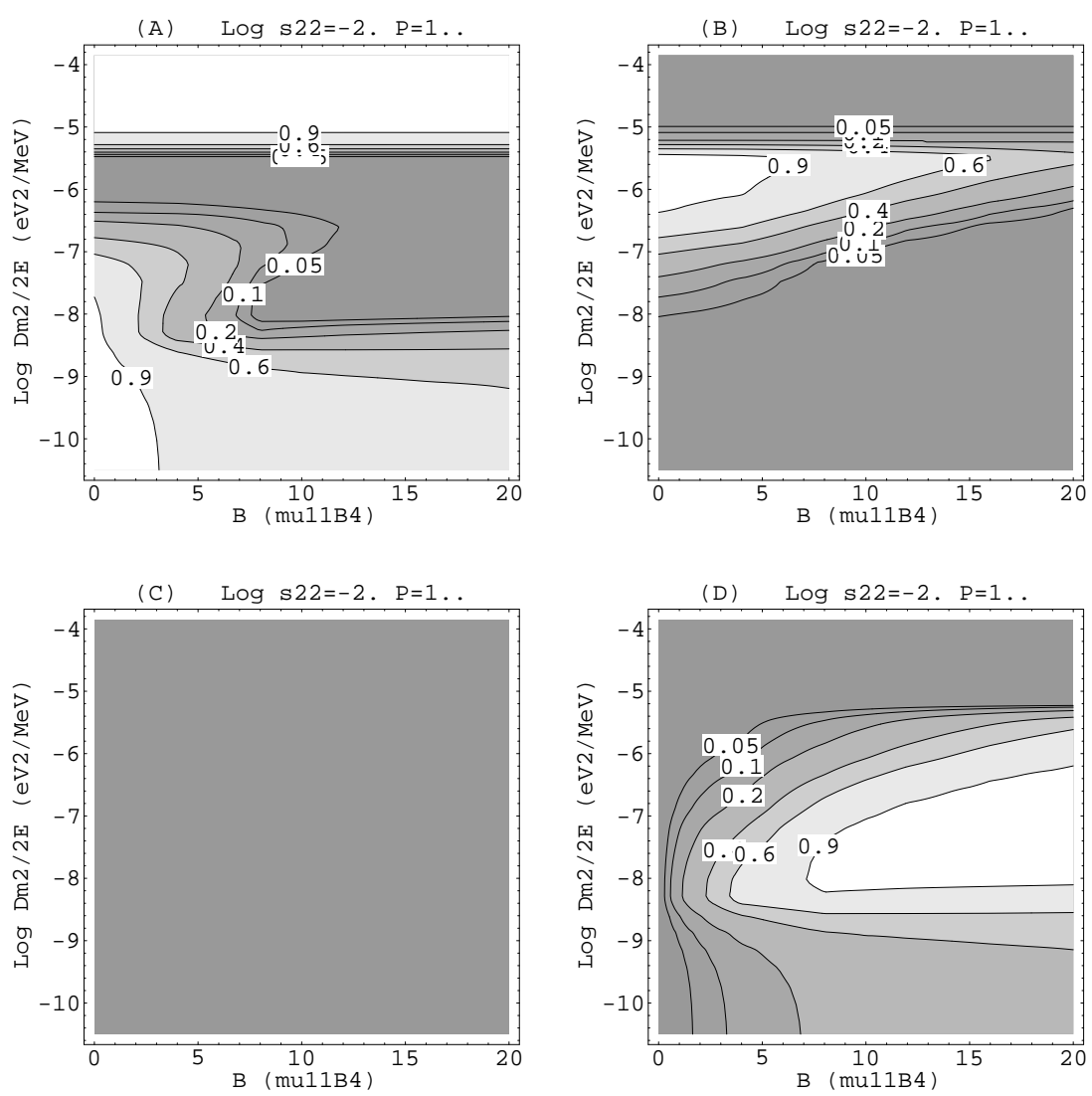

Figure 2: Probabilities at Earth for a neutrino created at the solar center as a function of the magnetic field taken at the bottom of the convective zone $\left(B\left(0.7 r_{0}\right)\right)$, for a fixed mixing angle $\left(s_{2}^{2}=0.01\right)$. Respectively A) $P_{\nu_{e} \nu_{e}}$, B) $P_{\nu_{e} \nu_{\mu}}$, C) $P_{\nu_{e} \overline{\nu_{e}}}$, D) $P_{\nu_{e}, \overline{\nu_{\mu}}}$. 

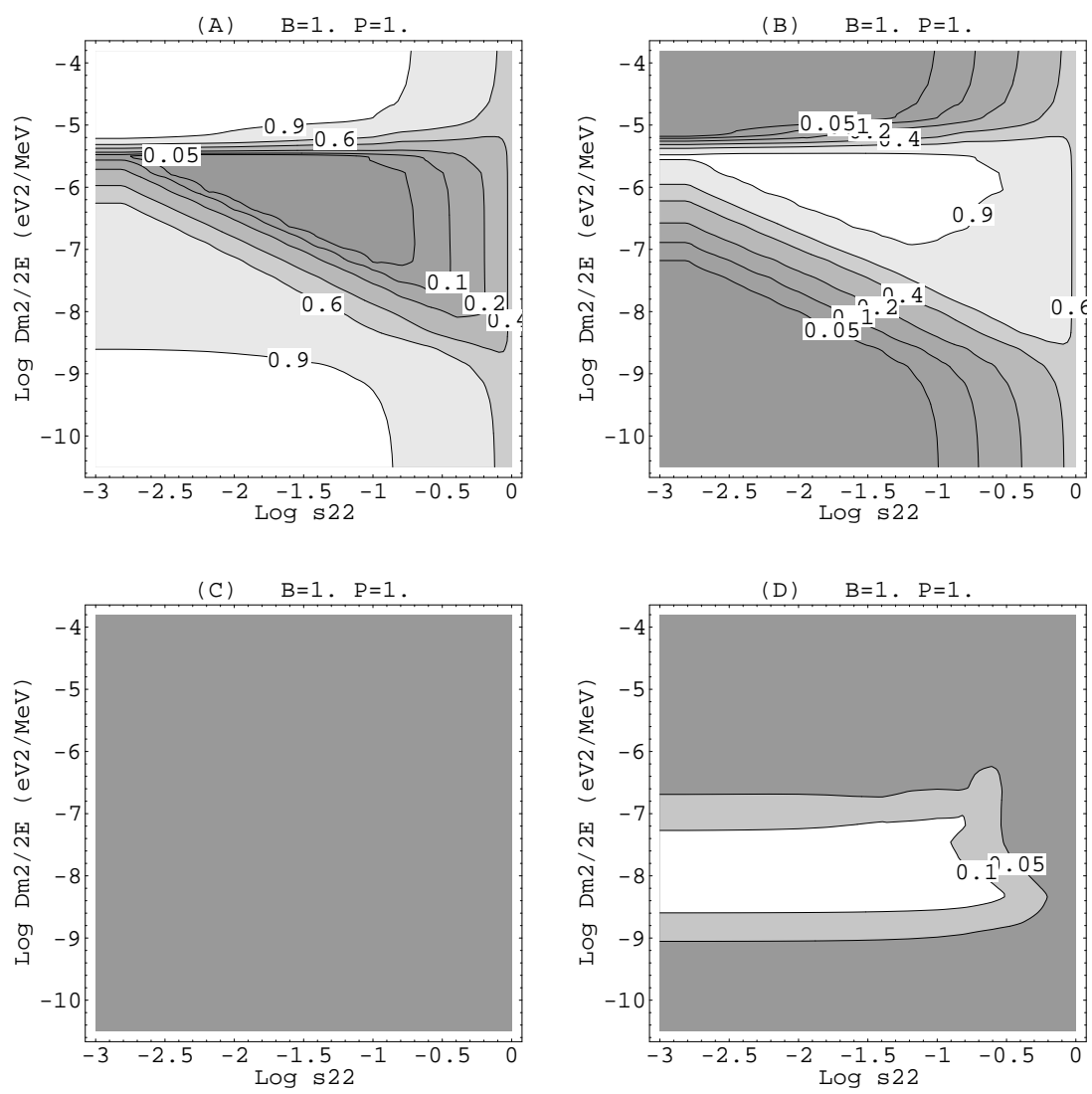

Figure 3: As previous figure. Probabilities as a function of $s_{2}^{2}$ for moderate magnetic field $\left(\mu B_{0.7}=1.0 \mu_{11} B_{4} \equiv 10^{-11} \mu_{B} 10^{4} \mathrm{G}\right)$. 

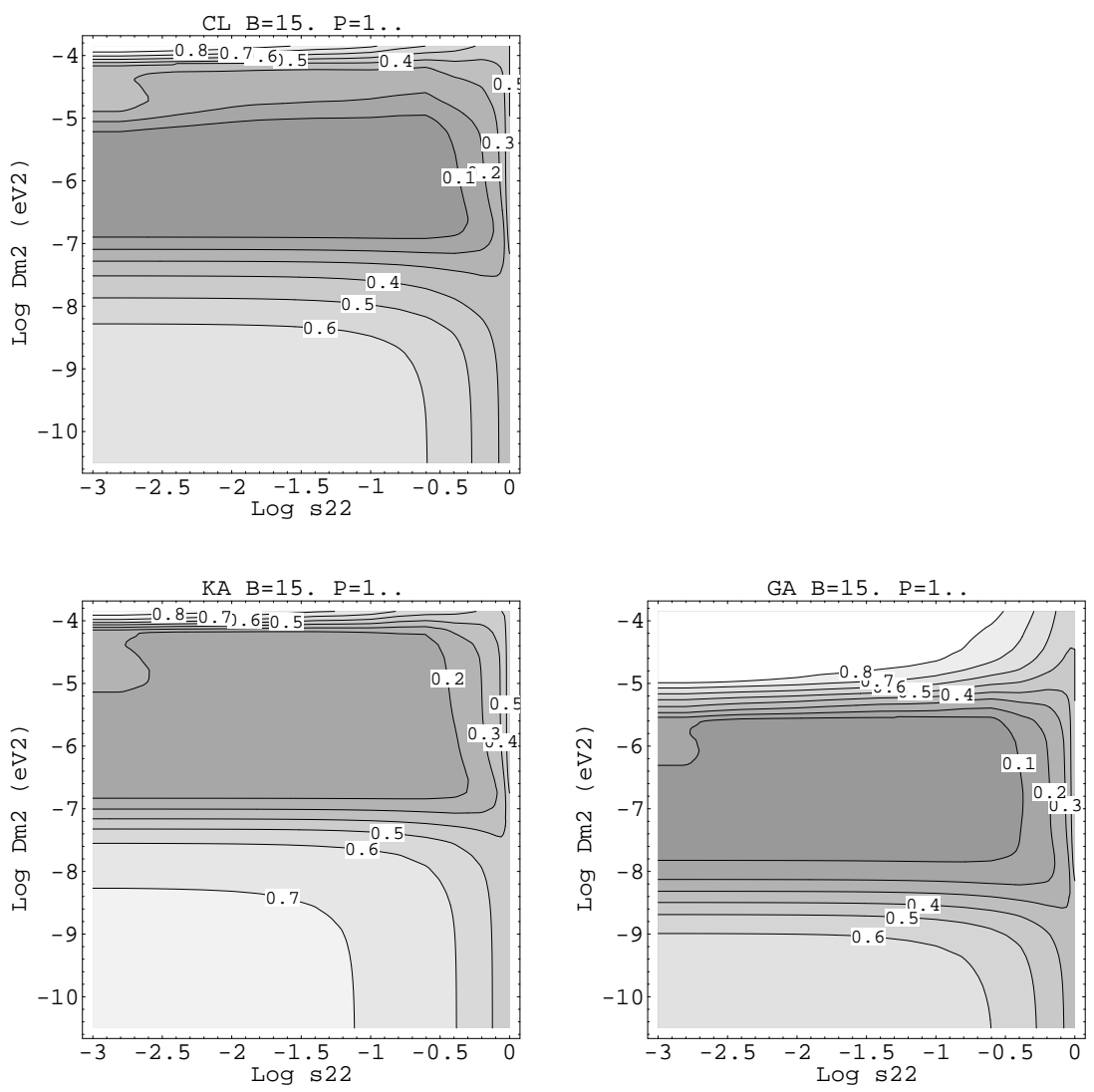

Figure 4: Relative signal in absence of noise $(P=1)$ at the different experiments: $(\mathrm{A})$ Homestake, (B) Kamiokande, (C) Gallium, as a function of the mixing angle ( $\log s_{2}^{2}$, horizontal scale) and squared mass differences $\left(\log \Delta m^{2}\left(\mathrm{eV}^{2}\right)\right.$, vertical scale). The magnetic field is fixed to be $\mu B=15 \mu_{11} B_{4}$ at the bottom of the convective zone $\left(r=0.7 R_{\odot}\right)$ 

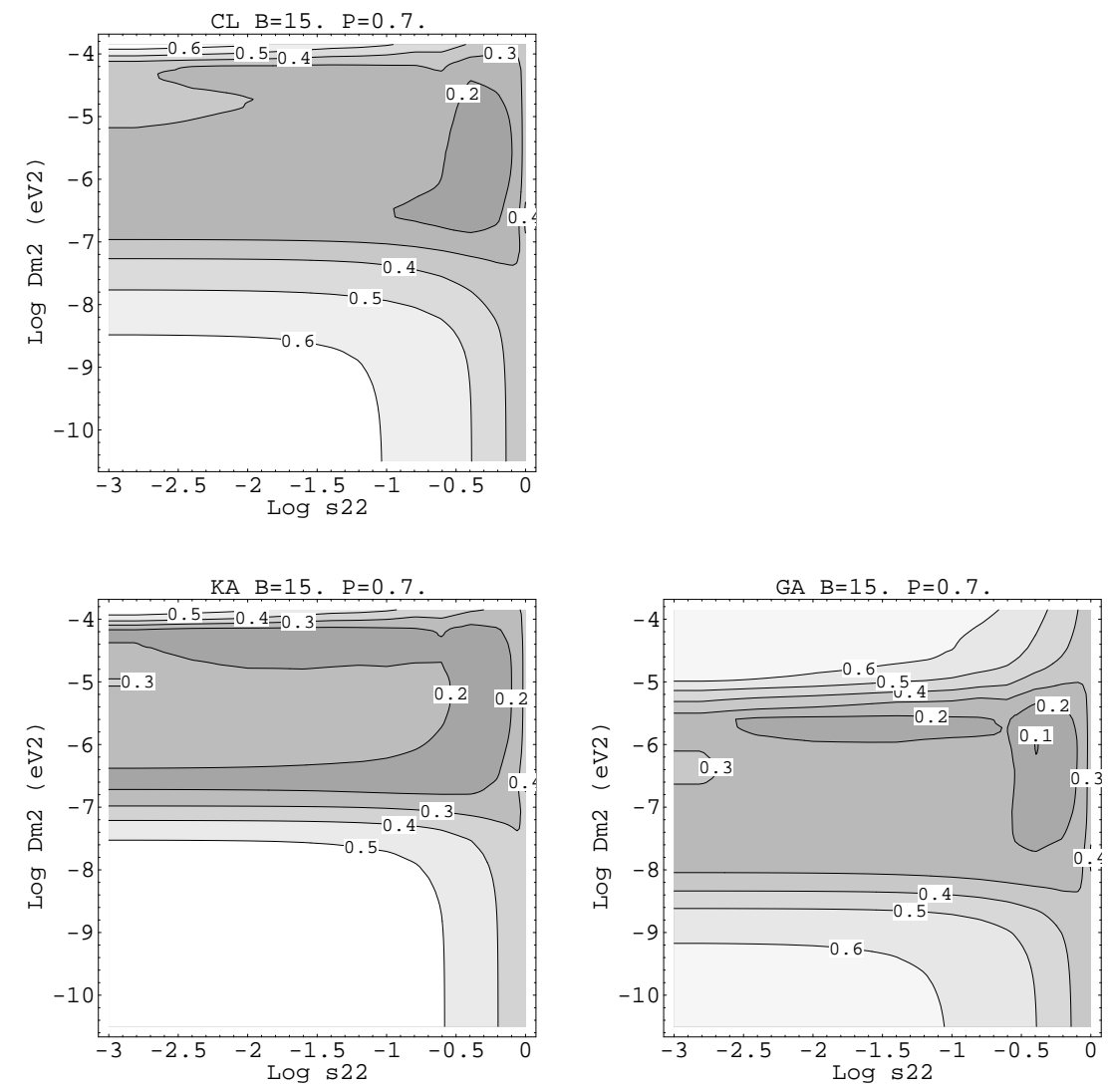

Figure 5: The same as Fig.(四) but in presence of noise $(P=0.7$, see Table 1). 
(A) $\mathrm{B}=1 . \mathrm{P}=1.00$.

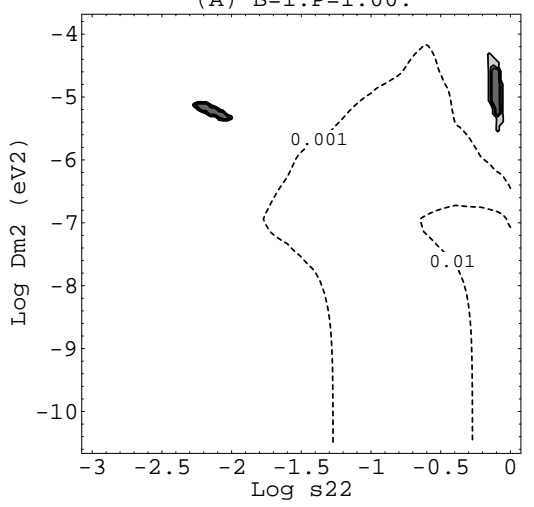

(C) $\mathrm{B}=8 \cdot \mathrm{P}=1.00$.

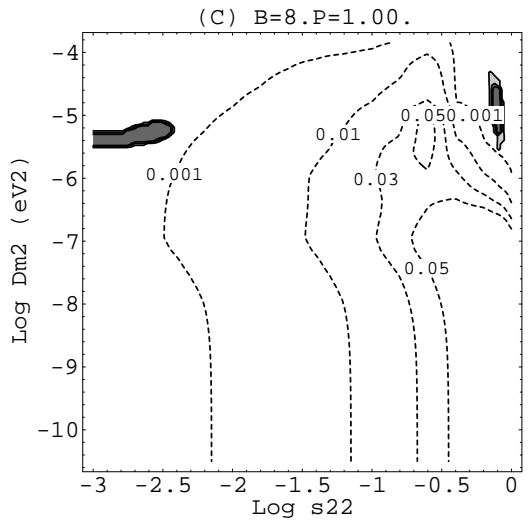

(B) $B=5 \cdot P=1.00$.

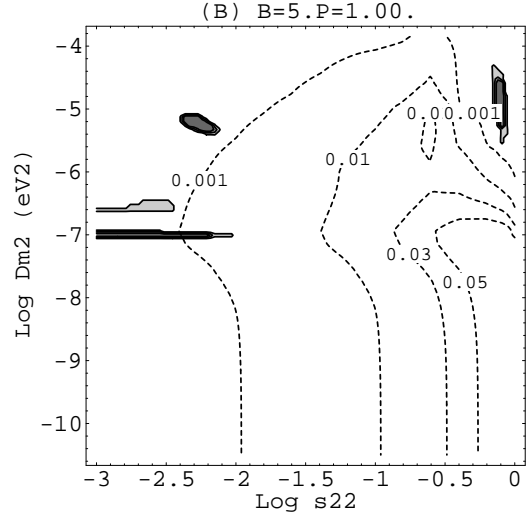

(D) $B=20 . P=1.00$.

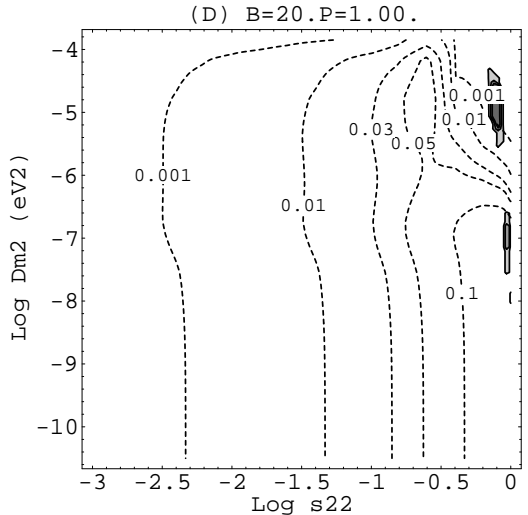

Figure 6: The parameter regions consistent with the Homestake, Kamiokande and combined Gallium experiments in absence of noise: $P=1.00$. (C.L. $=90 \%, 95 \%, 99 \%$ (from darkest to lighter shaded areas)). Plots (A,B,C,D) correspond respectively to values of the regular magnetic field at the bottom of the convective zone: $\mu B=1,5,8,20 \mu_{11} B_{4}$. The electron antineutrino averaged probability, Eq. (30), is represented by the dashed lines. Present Kamiokande bounds impose the additional restriction $P_{\nu_{e} \overline{\nu_{e}}}<0.05$ to the total rate allowed regions. 

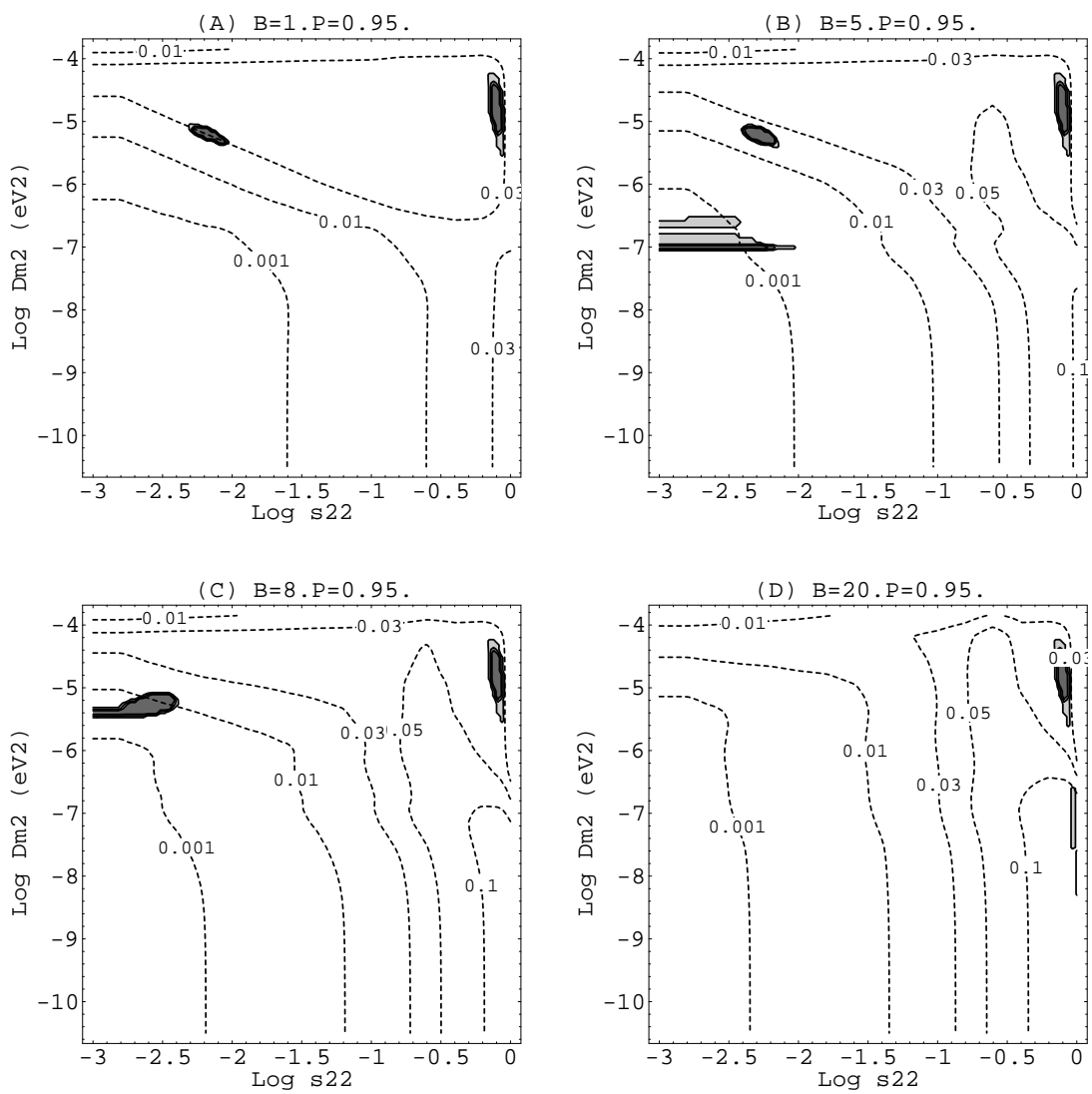

Figure 7: The parameter regions consistent with the Homestake, Kamiokande and combined Gallium experiments as Fig.(可) for $P=0.95$. 

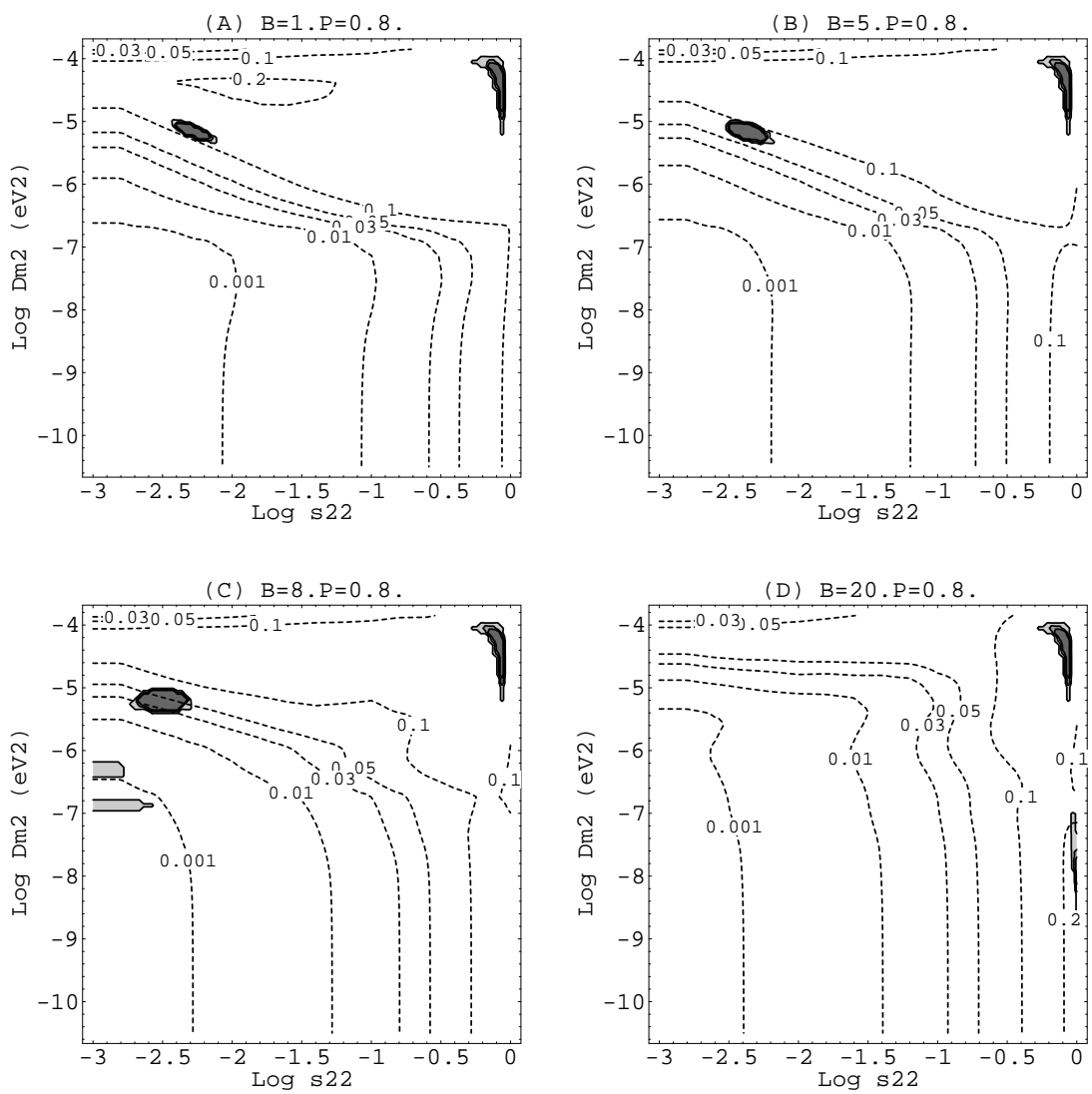

Figure 8: The parameter regions consistent with the Homestake, Kamiokande and combined Gallium experiments as Fig.(原). for $P=0.80$. 

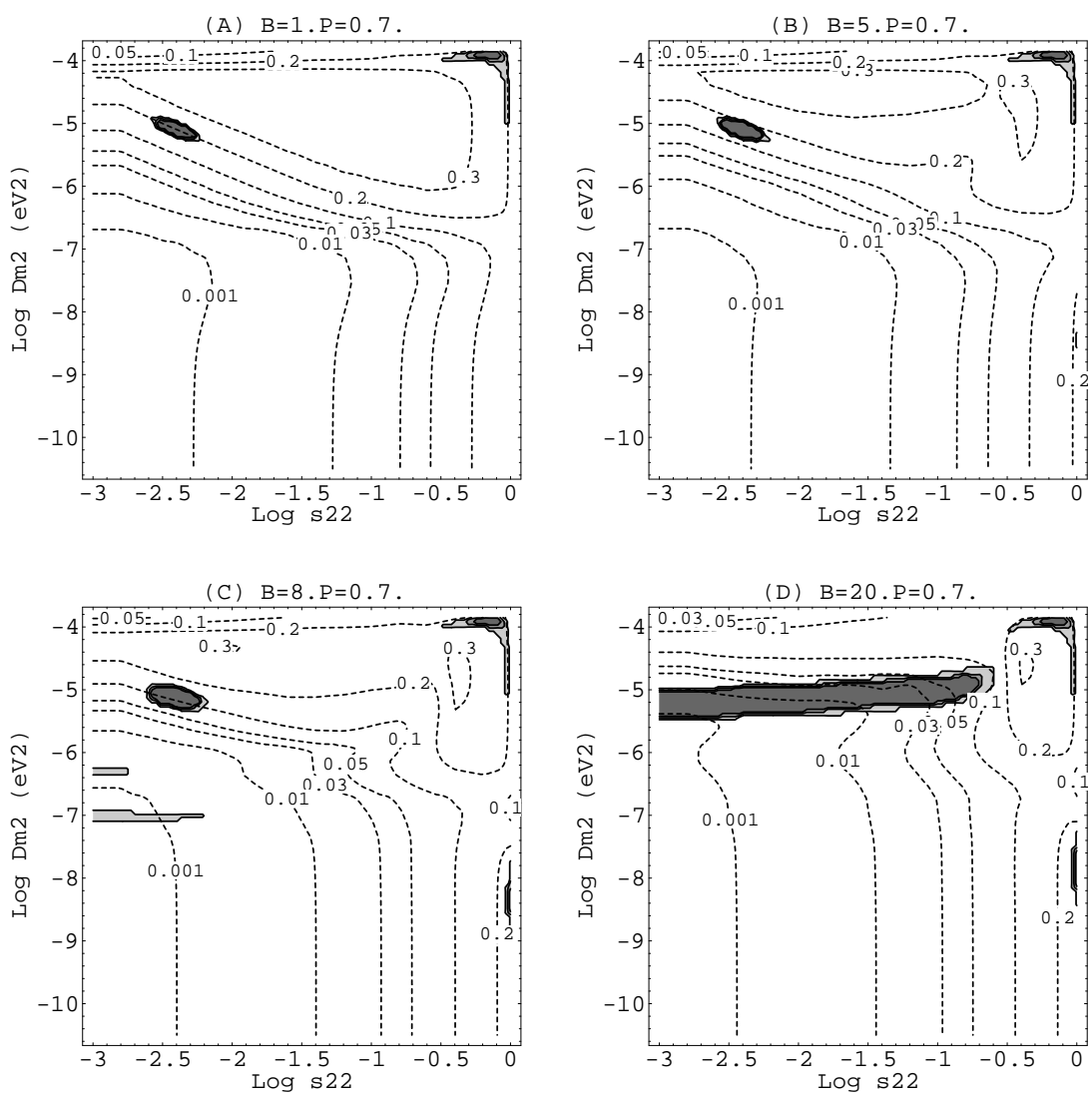

Figure 9: The parameter regions consistent with the Homestake, Kamiokande and combined Gallium experiments as Fig.(原). for $P=0.70$. 

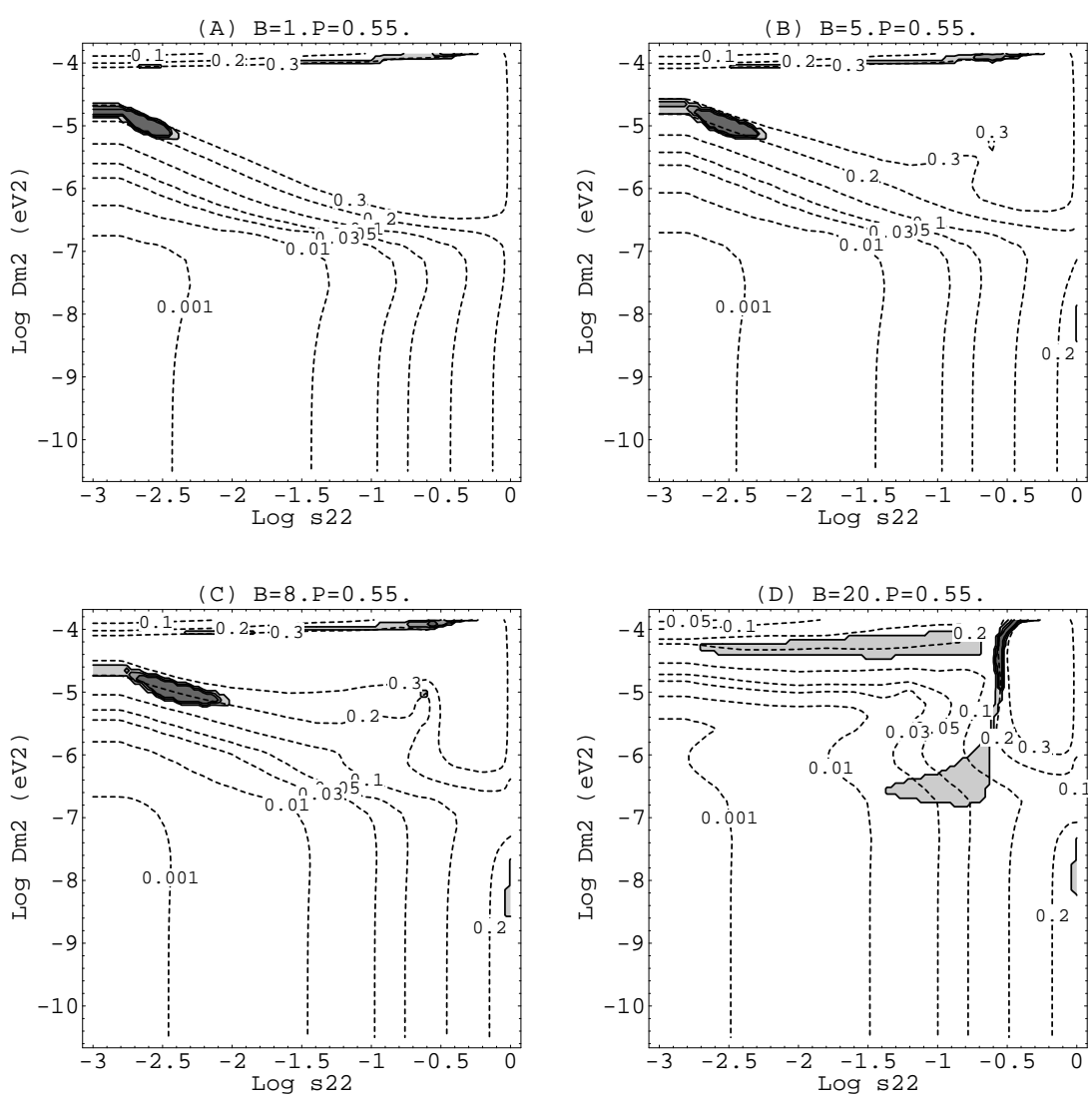

Figure 10: $\chi^{2}$ contour plots. C.L. $=90 \%, 95 \%, 99 \%$ (from darkest to lighter). for $P=0.55$. 